\title{
Thoracoscopic repair of congenital oesophageal atresia in a newborn: a case report
}

Michelle ON Yu1 ${ }^{1}$, FCSHK, FHKAM (Surgery), Patrick HY Chung ${ }^{1}$ *, FCSHK, FHKAM (Surgery),

Mabel Wong'2, FHKAM (Paediatrics), Anne Kwan³, FHKAM (Anaesthesiology), YE Chee³, FHKAM (Anaesthesiology), Kenneth KY Wong ${ }^{1}$, FCSHK, FHKAM (Surgery)

This article was published on 6 Dec 2021 at www.hkmj.org.
Hong Kong Med J 2022;28:73-5

\author{
${ }^{1}$ Department of Surgery, Queen Mary Hospital, Hong Kong \\ ${ }^{2}$ Department of Paediatrics and Adolescent Medicine, Queen Mary Hospital, Hong Kong \\ ${ }^{3}$ Department of Anaesthesiology, Queen Mary Hospital, Hong Kong \\ *Corresponding author: chungphy@hku.hk
}

\section{Case report}

In July 2021, a $1.5-\mathrm{kg}$ baby girl presented with polyhydramnios on antenatal ultrasound scan at 27 weeks that had resolved by 35 weeks. Physical examination showed no dysmorphic features. She was born by caesarean section at 35 weeks due to discordant growth in a dichorionic diamniotic twin pregnancy. She was intubated at birth but resistance was noted during orogastric tube insertion. Chest $\mathrm{X}$-ray revealed coiling of the gastric tube at the upper pouch of the oesophagus with the presence of intestinal gas, which is a classic feature of type $C$ oesophageal atresia (Fig 1). Echocardiogram revealed several cardiac anomalies including a ventricular septal defect, a moderate patent ductus arteriosus, and a large atrial septal defect.

Thoracoscopic repair of oesophageal atresia was scheduled for the next day following stabilisation. The patient was positioned in a semiprone position with the right chest slightly elevated. She was put on conventional ventilation without single lung ventilation, as per our practice. A camera port was inserted at the 5 th intercostal space and two further 5-mm working ports were inserted under direct vision. Pneumothorax of 3 to $4 \mathrm{~mm} \mathrm{Hg}$ was created using $\mathrm{CO}_{2}$. The azygous vein was cauterised and divided with monopolar diathermy and the tracheoesophageal fistula readily identified. This was closed by Weck Hem-o-lok (Teleflex; Wayne [PA], United States) and divided (Fig 2). The proximal oesophageal stump was identified and opened with scissors. End-to-end esophago-oesophagostomy was performed using single-layer interrupted 5/0 polydioxanone sutures (Ethicon; Bridgewater [NJ], United States). A 12-Fr chest drain was inserted at the end of the procedure. The operation was uneventful and completed in 145 minutes.

After surgery, the patient was managed in the neonatal intensive care unit according to our usual protocol. On day 1 after surgery she developed sudden profound desaturation with $\mathrm{CO}_{2}$ retention when the endotracheal tube was secured at $8 \mathrm{~cm}$

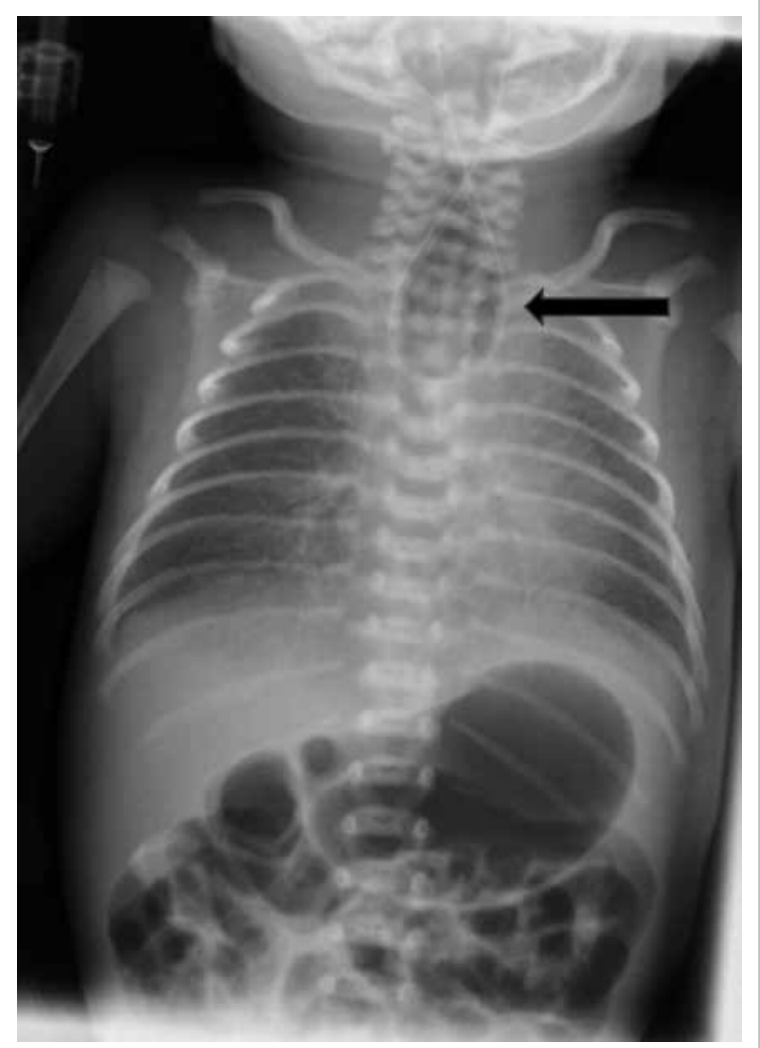

FIG I. A I.5-kg baby girl delivered at 35 weeks gestation. Plain radiograph taken at birth showing the orogastric tube (arrow) coiled at the proximal oesophageal pouch with distal bowel gas

from the upper lip. Bedside bronchoscopy showed a tracheal pouch (fistula remnant) close to the tip of the endotracheal tube. The tip completely entered the pouch with minimal advancement of the endotracheal tube. The endotracheal tube was then withdrawn to $7.5 \mathrm{~cm}$ from the upper lip and no further desaturation was noted.

A contrast swallow study on day 18 after surgery showed an intact anastomosis. The patient 


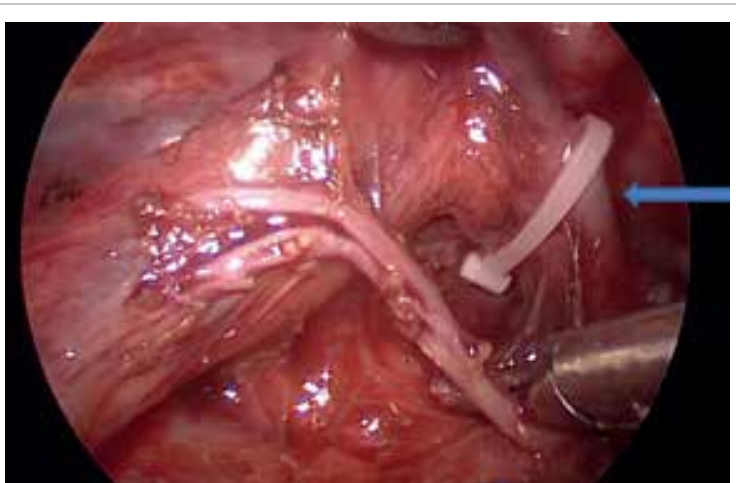

FIG 2. The same $1.5-\mathrm{kg}$ baby girl delivered at 35 weeks gestation. Surgical photograph showing the clipping of the tracheoesophageal fistula (arrow)

was extubated successfully on day 19 after surgery. Bolus feeding via a feeding tube was established after extubation, and oromotor training was commenced. She was transferred back to the referring hospital for management of her cardiac disorders. At 6 weeks after surgery, she had good weight gain with full oral feeding and no clinical gastroesophageal reflux.

\section{Discussion}

The introduction of minimally invasive surgery has undoubtedly revolutionised the treatment of many surgical disorders. The role of minimally invasive surgery in common neonatal procedures such as inguinal hernia repair and pyloromyotomy is well established. However, this operative approach in more complex procedures is still limited by various factors including equipment size, surgical expertise, and perioperative support.

Among all the neonatal operations, repair of oesophageal atresia is one of the most challenging. In addition to the need for meticulous surgical skill, adequate support from a dedicated neonatal intensive care unit and expert paediatric anaesthetists are equally important. The first successful thoracoscopic repair of oesophageal atresia was reported in 1999. A subsequent international multicentre study published 15 years ago further confirmed that thoracoscopic repair of oesophageal atresia was at least as good as traditional thoracotomy. ${ }^{2}$ However, this operative approach is still not widely practised.

In our unit, we started to perform thoracoscopic surgery in 2007. In our early experience reported in $2012,^{3}$ we selected patients with a reasonably large body size for minimally invasive surgery. With the accumulation of neonatal operative experience, we became confident performing these operations on smaller-sized babies. Prior to our case, Son et $\mathrm{al}^{4}$ published their experience in thoracoscopic repair of oesophageal atresia in babies $<2 \mathrm{~kg}$, and Rothenberg ${ }^{1}$ reported a successful experience in a $1.2-\mathrm{kg}$ baby. We believe that thoracoscopic repair of oesophageal atresia in neonates can be performed safely in experienced centres with proper case selection.

Traditionally, the Spitz classification has been considered the prognostic indicator. Neonates with oesophageal atresia with birth weight $<1.5 \mathrm{~kg}$ and major cardiac anomalies (as in our patient) are predicted to have only a $50 \%$ survival rate with a traditional open surgical approach. However, advances in surgical skills, neonatal care, and anaesthesia combined with the ability to optimise the surgical approach have resulted in improved surgical outcomes and consequent survival rates. ${ }^{1,4}$

The challenges faced in this operation included the patient's small size and presence of cardiopulmonary complications before and during surgery. The pneumothorax created during thoracoscopy may compress the lung causing difficulty in ventilation and compromise cardiac function, further increasing the complexity of anaesthesia. We overcame this by limiting the pressure to 3 to $4 \mathrm{~mm} \mathrm{Hg}$, resulting in less operative space but better patient tolerance. In our opinion, the reduced working space is not a major hurdle for a surgeon competent in minimally invasive surgery. In a baby with congenital heart disease, an anaesthetist who has experience in neonatal thoracic surgery is essential for optimum intra-operative management.

Holcomb et $\mathrm{al}^{2}$ demonstrated in a multiinstitutional study that there were no significant differences in reported postoperative complication rates between minimally invasive surgery and open repair. A minimally invasive approach has the additional benefits of smaller wounds and less pain. More specifically, thoracoscopic repair allows for clearer magnification. Although $\mathrm{CO}_{2}$ pneumothorax will compress the right lung during surgery, its effect is significantly less than that of manual compression during open surgery. Long-term studies of musculoskeletal problems also reveal a superior outcome for thoracoscopic surgery. ${ }^{1}$

In conclusion, we report a successful thoracoscopic repair of oesophageal atresia in a high-risk neonate with very low birth weight. To the best of our knowledge, this is the smallest baby with oesophageal atresia in Hong Kong to have this operation. While proper case selection to ensure patient safety remains the top priority, small body size should not preclude a thoracoscopic surgical approach. The combined efforts and advances in surgery, anaesthesia and neonatal care are key to success.

\section{Author contributions}

All authors contributed to the concept or design of the study, acquisition of the data, analysis or interpretation of the 
data, drafting of the manuscript, and critical revision of the manuscript for important intellectual content. All authors had full access to the data, contributed to the study, approved the final version for publication, and take responsibility for its accuracy and integrity.

\section{Conflicts of interest}

As the editor of the journal, KKY Wong was not involved in the peer review process for this article. Other authors have no conflicts of interest to disclose.

\section{Funding/support}

This study received no specific grant from any funding agency in the public, commercial, or not-for-profit sectors.

\section{Ethics approval}

The patient was treated in accordance with the Declaration of Helsinki. The patient's parents provided informed consent for all treatments and procedures and provided consent for publication.

\section{References}

1. Rothenberg SS. Thoracoscopic repair of esophageal atresia and tracheo-esophageal fistula in neonates: evolution of a technique. J Laparoendosc Adv Surg Tech A 2012;22:195-9.

2. Holcomb GW 3rd, Rothenberg SS, Bax KM, et al. Thoracoscopic repair of esophageal atresia and tracheoesophageal fistula: a multi-institutional analysis. Ann Surg 2005;242:422-8.

3. Huang J, Tao J, Chen $\mathrm{K}$, et al. Thoracoscopic repair of oesophageal atresia: experience of 33 patients from two tertiary referral centres. J Pediatr Surg 2012;47:2224-7.

4. Son J, Jang Y, Kim W, et al. Thoracoscopic repair of esophageal atresia with distal tracheoesophageal fistula: is it a safe procedure in infants weighing less than $2000 \mathrm{~g}$ ? Surg Endosc 2021;35:1597-601. 